\title{
Multimodality imaging and interventional management of a complex congenital vascular malformation
}

\section{Aadil Ahmed, MB BCh, FCRad (Diag)}

Visser, Erasmus, Vawda and Partners, Port Elizabeth

\section{Introduction}

Hepatic vascular lesions are not an uncommon finding in children, and represent the most common benign liver tumours to present in infancy. We present a case of a complex vascular malformation with an intrahepatic component suggestive of a venous/arteriovenous malformation as well as a large extrahepatic lesion. The extrahepatic mass was present in both sub- and supra-diaphragmatic locations, with features of a congenital haemangioma.

In view of the clinical presentation and different imaging appearances, this case was felt to be interesting in its radiological workup, management and eventual unusual pathology.

\section{Case report}

A term neonate presented a few days after birth with worsening respiratory distress that required ventilation. A left-sided pleural effusion had been treated in utero by means of a pleuro-amniotic shunt at 27 weeks' gestation. The ultrasound and chest X-ray done shortly after birth showed a left pleural effusion and a lobulated, moderately vascular

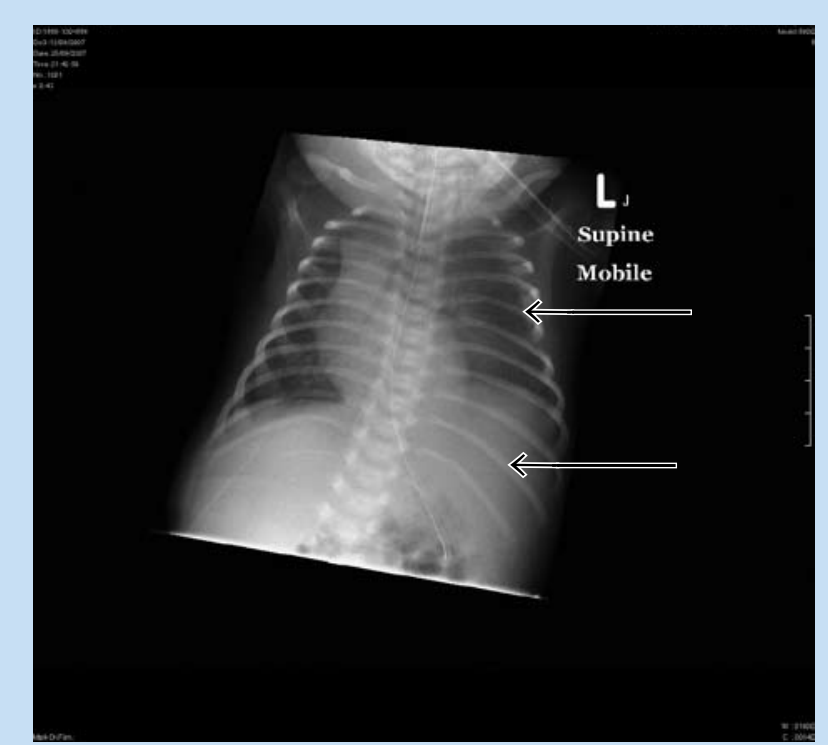

Fig. 1a. CXR demonstrates left subphrenic mass (arrow) displacing nasogastric tube in stomach inferiorly. Left-sided effusion present (arrowhead).

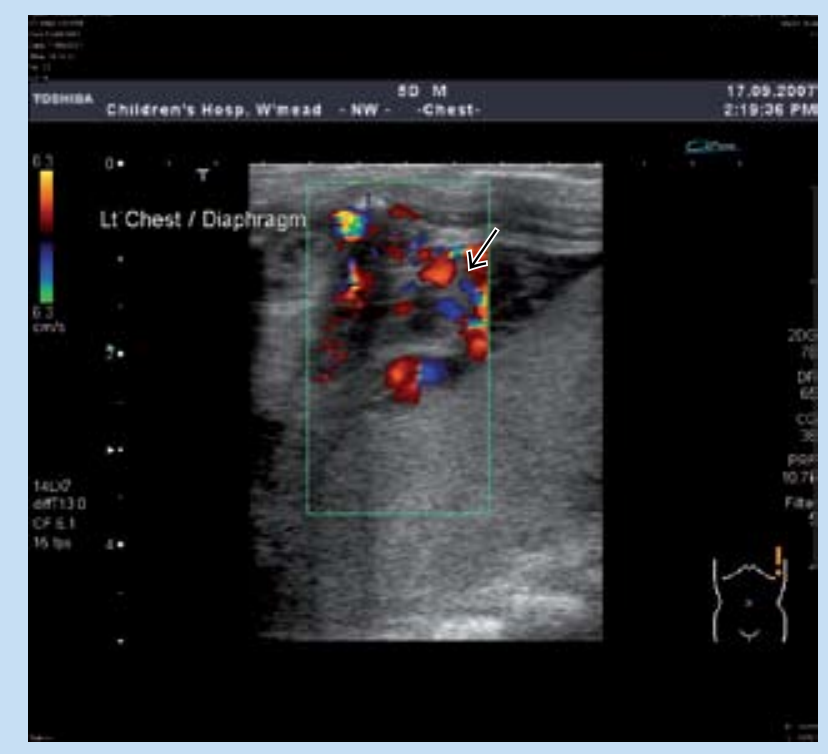

Fig. 1b. Colour Doppler ultrasound shows vascular malformation (arrow) displacing spleen inferiorly.

thoraco-abdominal mass displacing the spleen and kidney inferiorly (Fig. 1). The patient did not have any cardiovascular compromise, and echocardiography demonstrated normal anatomy and function.

Subsequent computed tomography (CT), magnetic resonance imaging (MRI) and magnetic resonance angiography (MRA) studies further demonstrated the extent, hepatic involvement, appearance and vascularity of the lesion. A well defined, predominantly solid, lobulated mass exhibiting diffuse early vascular enhancement was seen, which on delayed imaging remained dense with increased centripetal opacification. The mid portion of the left hemidiaphragm was not clearly defined (Fig. 2).

The multiple feeding arteries were best demonstrated on the contrast-enhanced MRA, showing a large artery arising from the abdominal aorta, adjacent to the coeliac axis (Fig. 3). There was also significant supply seen from a branch of the left hepatic artery, as well as intercostal arteries and the left internal mammary artery. Venous drainage was directly into the IVC via a large aberrant vein running parallel to the left hepatic vein, as well as into the left renal vein (Fig. 4). In addition, multiple serpentine vessels were seen surrounding the lesion, with some noted to drain into the spinal epidural venous plexus, as well as left subclavian vein. The possibility of this being an extralobar pulmonary sequestration was considered at this stage.

An angiogram was thereafter performed to further characterise the vascularity, as well as allow embolisation of the lesion prior to surgery (Fig. 5). 


\section{CASE REPORT}

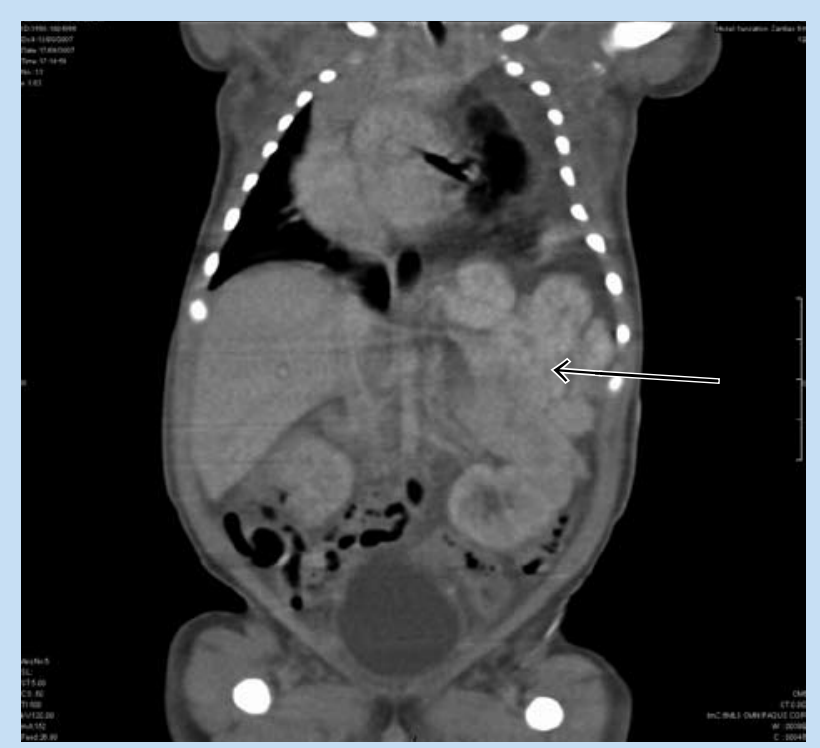

Fig. 2a. Coronal CT image: enhancing, lobulated mass (arrow), displacing intra-abdominal viscera and reactive left pleural effusion.

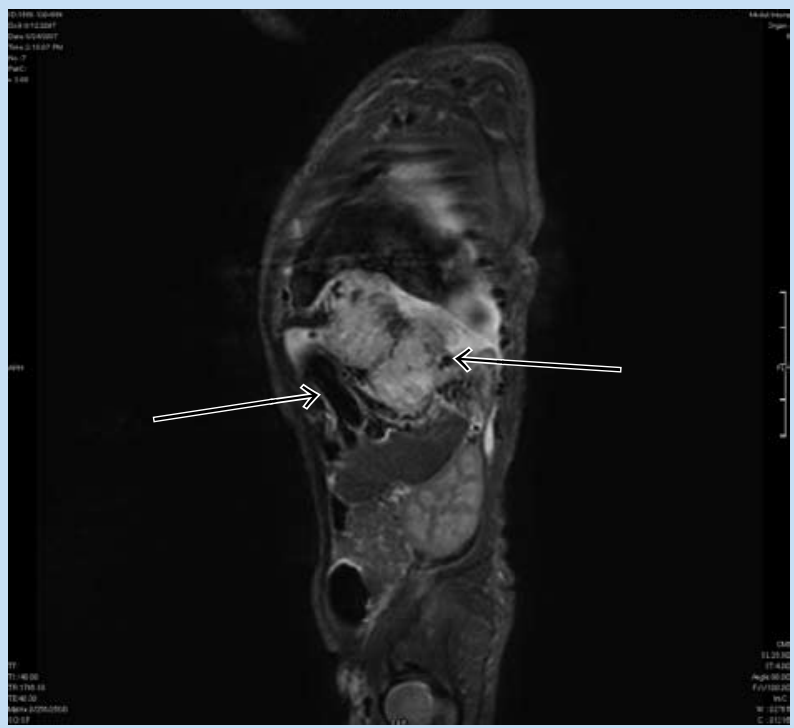

Fig. 2b. Hyperintense mass on sagittal T2 fat suppression sequence. Prominent vascular flow voids seen (arrows). The diaphragm is poorly outlined.

Branches arising from the left hepatic artery supplying the vascular malformation were successfully occluded using Onyx (Micro Therapeutics Inc.). The anomalous, tortuous vessel arising from the aorta was seen to supply the solid component related to the left hemidiaphragm. Some of the former's branches were embolised using Hepasphere embospheres (Biosphere Medical) and n-butyl cyanoacrylate.

At surgery the following day, the supra- and sub-diaphragmatic vascular mass was excised, and a partial left hepatectomy was performed.

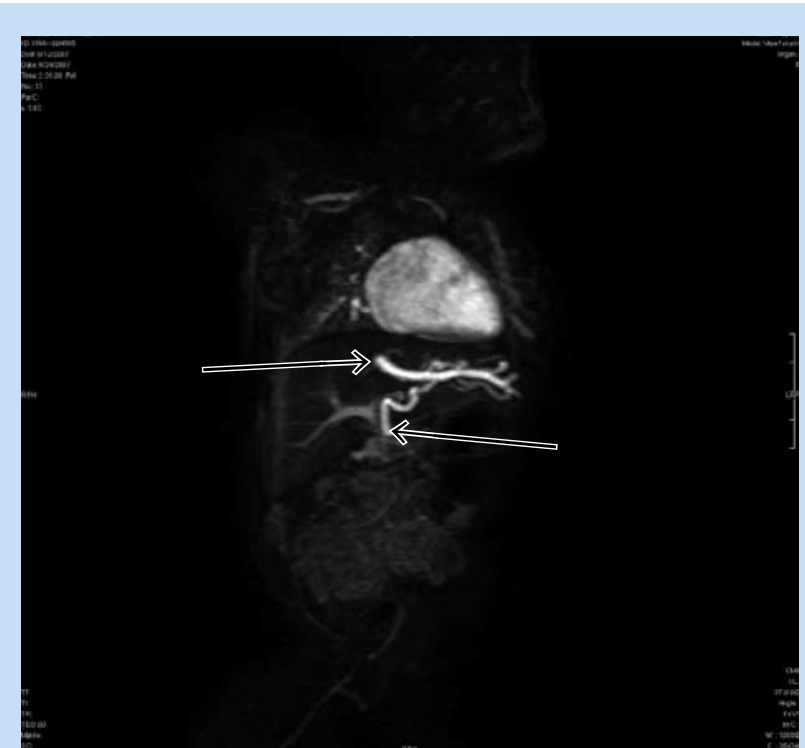

Fig. 3. Coronal MIP image from contrast-enhanced MRA study. The dominant arterial supply from the abdominal aorta and left hepatic artery are shown (arrows).

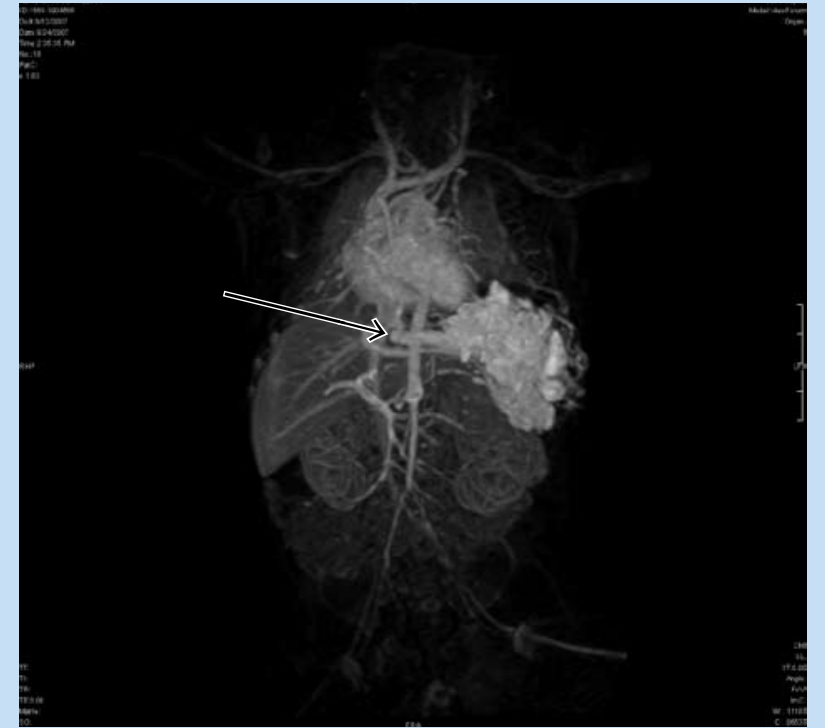

Fig. 4. Coronal MIP image. Delayed phase of contrast-enhanced MRA. Venous drainage to the IVC is shown (arrow).

Histological analysis confirmed the hepatic lesion to be a mixed vascular malformation. The diaphragmatic mass probably represented a vascular malformation with a predominantly capillary component; however, some features suggestive of a congenital haemangioma were present. Glucose transporter-1(Glut-1) stains were negative in all lesions.

The patient made an uneventful recovery and, at the time of this report (4 months later), is symptom-free, with the follow-up MRI showing no residual mass or vascular abnormality (Fig. 6). 


\section{CASE REPORT}

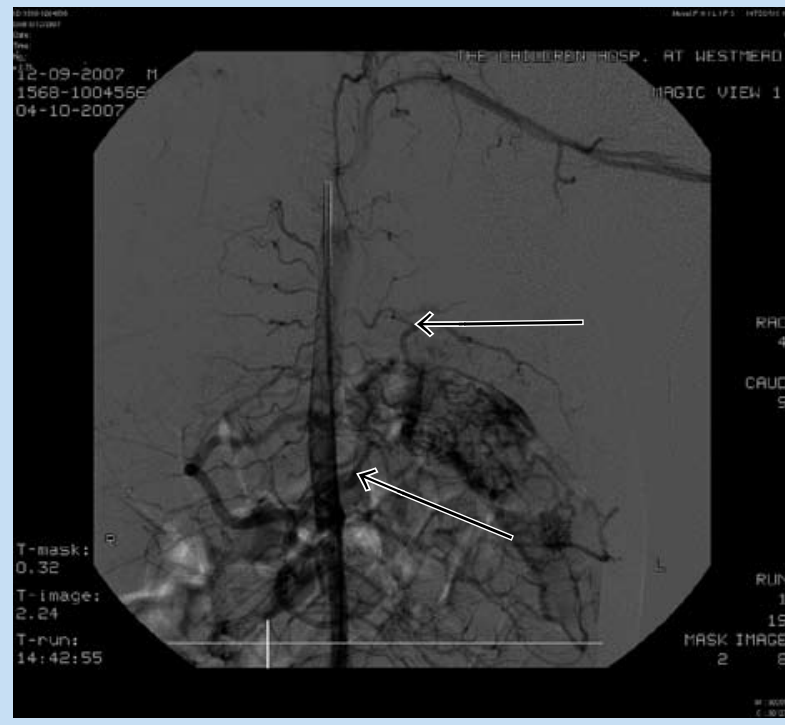

Fig. 5a. Flush aortogram demonstrating extensive arterial supply, including branches from intercostal arteries (arrows).

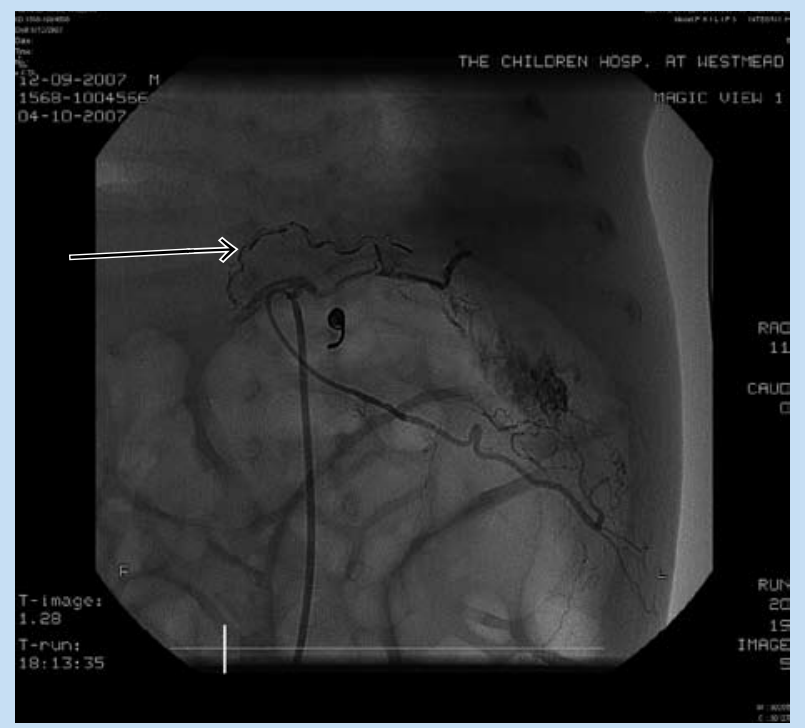

Fig. 5b. Arteriogram done during the embolisation. Onyx is seen in branches arising from the left hepatic artery (arrow). An embolisation coil was also used to occlude one of the feeder vessels. Dense contrast blush is seen in the solid component of the malformation (arrowhead).

\section{Discussion}

Based on their biological and clinical behaviour, vascular anomalies can be broadly divided into two groups: vascular tumours and vascular malformations. Histologically identified lesions belonging to both these groups were present in our patient. The extrahepatic mass was confirmed to be a congenital haemangioma, a clinical entity distinct from the more common haemangioma of infancy. These congenital tumours bear some resemblance to haemangiomas of infancy but, unlike these, are fully developed and do not undergo any post-natal enlargement. Congenital

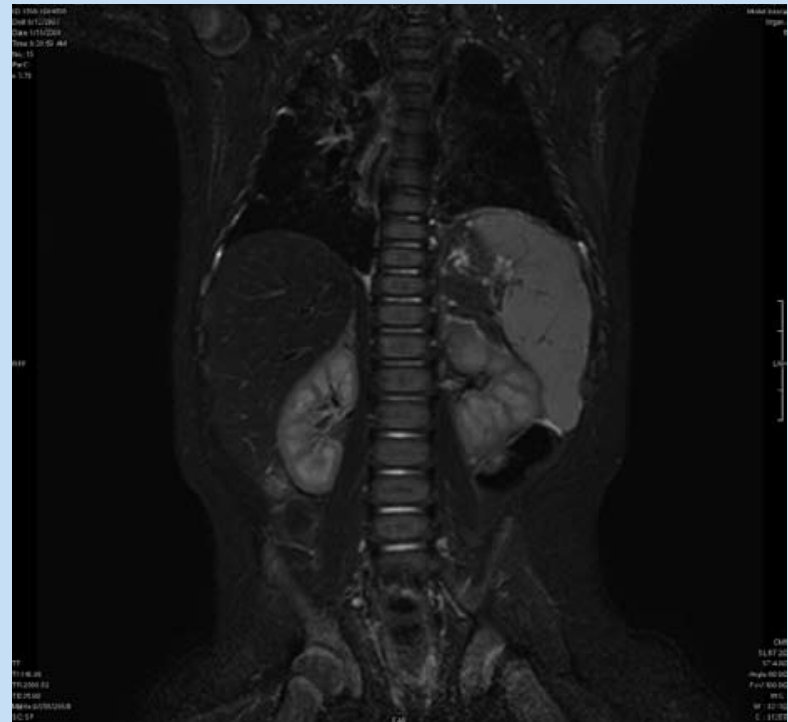

Fig. 6. Coronal MRI image, four months after intervention and surgery, demonstrating no residual malformation and near-normal anatomy.

haemangiomas also stain negative for glucose transporter-1 protein (Glut-1), a marker of haemangioma of infancy.

There is a further differentiation of congenital haemangiomas into rapidly involuting congenital haemangiomas ( $\mathrm{RICH}$ ) and non-involuting congenital haemangiomas (NICH). ${ }^{1}$ The haemangioma in our patient probably falls within the NICH category in view of the lack of significant regression in size although, admittedly, intervention occurred very early in the patient's life, and might have affected the natural history.

Vascular malformations are congenital lesions of vascular dysmorphogenesis, are always present at birth, and enlarge in proportion to the growth of the child. Vascular malformations can be divided on the basis of vascular channel type (capillary, lymphatic, venous, arterial or combined) or by flow (slow or fast). ${ }^{2}$ On angiography, reasonably fast flow and shunting was demonstrated in the intrahepatic malformation. Histological inspection showed arterial components, which would also support this lesion being a high-flow malformation.

Multiple modalities can be used to examine vascular lesions, with MRI and ultrasound being the most useful non-invasive tools, providing important information on disease extent and involvement of vital structures. Imaging also helps to characterise lesions and, with MRA, the vascular supply and drainage can specifically be assessed. Gadoliniumenhanced images have been advocated in differentiating between lowflow vascular malformations and venous malformations. Doppler characteristics of vascular malformations are also helpful in differentiating low- from high-flow vascular malformations. ${ }^{3}$

Embolisation is an important component in the treatment of arteriovenous malformations, and is considered the most effective treatment/ palliation of arteriovenous malformations. ${ }^{4}$

In our patient, owing to the complexity of the lesion, it was accepted that embolisation alone would not achieve definitive treatment. The goals of the embolisation here were to diminish hepatic arterial and collateral flow as best as possible to facilitate subsequent surgical resection. 


\section{CASE REPORT}

In this regard, the procedure was considered successful as it significantly decreased vascularity.

This multidisciplinary approach to the assessment and treatment of haemangiomas and vascular malformations is vital for the effective management and care of such patients.
Powell J. Update on haemangiomas and vascular malformations. Curr Opin Pediatr 1999; 11: 457-463.

2. Ashcraft KW, Holcomb GW, Murphy JP. Pediatric Surgery, 4th ed. Philadelphia: Elsevier Saunders, 2005: 1038-1039.

3. Donnelly LF, Adams DM, Bisset GS. Vascular malformations and haemangiomas: A practical approach in a multidisciplinary clinic. Am J Roentgenol 2000; 174: 597-608.

4. Yakes WF, Rossi P, Odink H. Arteriovenous malformation management. Cardiovasc Intervent Radiol 1996; 19: 65-71.w

\section{First RSSA short course on Current Ethical Issues in Radiology: 23 January 2010}

As a result of many requests from RSSA members as well as the rapidly changing professional, social, technical and political environment, this workshop was established to meet the important challenges facing radiologists and other significant role players. Topics pertinent to the daily practice of radiology were presented.

\section{Ethical considerations in cochlear implant patients}

Dr Derrick Wagenfeld (Head of the Tygerberg and Stellenbosch University Cochlear Implant Unit gave a fascinating insight into the technology, the cost implications, the vociferous opposition to the procedure (paradoxically from the deaf themselves), and the significant challenges facing those tasked with deciding who should be considered for the procedure. The role of the radiologist in the critical decision process was presented, and Dr Wagenfeld's eloquent delivery left the audience in no doubt why he was recognised as the best debater in his matric year at the age of 16 . This presentation inspired several immediate registrations for the forthcoming first RSSA/Leuven ear imaging course to be held at the Spier Estate.

\section{Self referral - a moral hazard}

Dr Clive Sperryn (President of the RSSA) presented an address on the moral hazard of self-referral which is prevalent in the fee-for-service environment. Despite the arguments in favour of self-referral advanced by lobby groups, research by independent neutral organisations confirms that self-referral leads not only to increased utilisation (by 1.7 - 7.7 times) but also to higher imaging costs (5 - 104\%) and more expensive treatment. Increased cost of care, where the cost of care outstrips the ability of a population to afford it, has lead directly to the current healthcare cost crisis in the USA. Self-referral has undesirable consequences that have been well documented in the USA, where \$16 billion per year is spent on unnecessary self-referred non-invasive diagnostic imaging. Utilisation is the way that insurance companies determine their premiums and, if utilisation is artificially inflated by self-referrals, insurance is pushed even further out of the reach of many people across the country.

Radiologists are a referred-to speciality but are not immune from this moral hazard. Radiologists are prevented, as far as possible, from generating their own referrals; however, it is incumbent on every radiologist to avoid becoming a facilitator of self-referral by others and to refrain from overuse of contrast studies, inappropriate or unnecessary imaging or up-coding.

Any inappropriate administration of ionising radiation, whether owing to kickbacks, perverse incentives, ignorance, defensive medicine, overservicing or self referral, contributes to unnecessary radiation exposure.

\section{Paediatric imaging: the ethics of radiation exposure}

Dr Savvas Andronikou (President of the College of Radiologists of South Africa) delivered an enthusiastic address in his unique, highly entertaining and inimitable style, challenging the audience as a serious message was delivered. Radiologists were reminded of the significantly increased radiation risks to the vulnerable paediatric population and implored to seriously consider whether each exposure was necessary and whether an alternate form of imaging such as ultrasound, MRI or nuclear medicine might not be more appropriate. Radiologists were also challenged to consider whether they were competent to perform studies on children using the lowest possible dose, whether the equipment used was up to the task in terms of dose reduction techniques such as pulsed fluoroscopy, and whether the settings had actually been modified for the paediatric examination. Do radiologists use screen capture techniques to record studies rather than full exposures? How often do radiologists contact the referring clinician to advise against an inappropriate examination and recommend an alternative investigation?

\section{Patient confidentiality in clinical practice: Is HIV status still relevant?}

Professor Keymanthri Moodley is head of the Bioethics Unit at Tygerberg and has served on the Research Ethics Committee for the past 10 years. It was a privilege to be addressed by a professional ethicist and to be guided through the minefield of patient confidentiality in the context of responsibilities both to the individual patient and society. The issue of HIV confidentiality was placed in an historical perspective, and AIDS exceptionalism highlighted. The presentation generated passionate discussion on HIV confidentiality, particularly in the context of healthworker safety and needle-stick injuries, with strong opinions from the floor and clear professional responses from Professor Moodley.

\section{Kick-backs and perverse incentives}

Dr Bates Alheit, the RSSA medical director, reviewed the definitions of kick-backs and perverse incentives: Any actions or business 